\title{
Correction to: An Angle-Insensitive Metamaterial Absorber Based on the Gravity Field Regulation
}

\author{
Xing-Liang $\operatorname{Tian}^{1} \cdot$ Hai-Feng Zhang ${ }^{1,2,3,4} \cdot$ Xin-Ru Kong $^{1}$ \\ Published online: 20 April 2021 \\ (c) Springer Science+Business Media, LLC, part of Springer Nature 2021
}

\section{Correction to: Plasmonics (2020) 15:517-523 https://doi.org/10.1007/s11468-019-01077-7}

Publisher's Note Springer Nature remains neutral with regard to jurisdictional claims in published maps and institutional affiliations.

The original version of this article unfortunately contained a mistake. We figure out that the captured grant number in the Funding section is incorrect. The correct Funding information is given below.

The original article has been corrected.

Funding This work was supported by the Open Research Program in China's State Key Laboratory of Millimeter Waves (Grant No. K201927) and Postgraduate Research \& Practice Innovation Program of Jiangsu Province (Grant No. KYCX19_0958).

The original article can be found online at https://doi.org/10.1007/ s11468-019-01077-7.

Hai-Feng Zhang

hanlor@163.com

1 College of Electronic and Optical Engineering \& College of Microelectronics, Nanjing University of Posts and Telecommunications, Nanjing 210023, China

2 National Electronic Science and Technology Experimental Teaching Demonstrating Center, Nanjing University of Posts and Telecommunications, Nanjing 210023, China

3 National Information and Electronic Technology Virtual Simulation Experiment Teaching Center, Nanjing University of Posts and Telecommunications, Nanjing 210023, China

4 State Key Laboratory of Millimeter Waves, Southeast University, Nanjing 210096, China 